\title{
Correction to: Missing Conflict of Interest statements in previously published articles
}

\author{
Waste Disposal \& Sustainable Energy Editorial Office ${ }^{1}$
}

Published online: 27 August 2021

(c) Zhejiang University Press 2021

\section{Correction to: Waste Dispos. Sustain. Energy \\ https://doi.org/10.1007/s42768-019-00001-3, https://doi.org/10.1007/s42768-019-00002-2, https://doi.org/10.1007/s42768-019-00003-1, https://doi.org/10.1007/s42768-019-00004-0,}

The original articles can be found online at https://doi.org/10.1007/ s42768-019-00001-3, https://doi.org/10.1007/s42768-019-000022, https://doi.org/10.1007/s42768-019-00003-1, https://doi.org/10. 1007/s42768-019-00004-0, https://doi.org/10.1007/s42768-01900005-z, https://doi.org/10.1007/s42768-019-00007-x, https://doi. org/10.1007/s42768-019-00008-w, https://doi.org/10.1007/s42768019-00009-9, https://doi.org/10.1007/s42768-019-00010-2, https:// doi.org/10.1007/s42768-019-00011-1, https://doi.org/10.1007/ s42768-019-00012-0, https://doi.org/10.1007/s42768-019-00013z, https://doi.org/10.1007/s42768-019-00014-y, https://doi.org/10. 1007/s42768-019-00015-x, https://doi.org/10.1007/s42768-01900017-9, https://doi.org/10.1007/s42768-019-00019-7, https://doi. org/10.1007/s42768-019-00020-0, https://doi.org/10.1007/s42768019-00022-y, https://doi.org/10.1007/s42768-019-00023-x, https:// doi.org/10.1007/s42768-019-00024-w, https://doi.org/10.1007/ s42768-019-00025-9, https://doi.org/10.1007/s42768-019-000268, https://doi.org/10.1007/s42768-019-00027-7, https://doi.org/10. 1007/s42768-019-00028-6, https://doi.org/10.1007/s42768-01900029-5, https://doi.org/10.1007/s42768-019-00030-y, https://doi. org/10.1007/s42768-019-00031-x, https://doi.org/10.1007/s42768019-00032-w, https://doi.org/10.1007/s42768-020-00033-0, https:// doi.org/10.1007/s42768-020-00035-y, https://doi.org/10.1007/ s42768-020-00036-x, https://doi.org/10.1007/s42768-020-00037w, https://doi.org/10.1007/s42768-020-00038-9, https://doi.org/10. 1007/s42768-020-00039-8, https://doi.org/10.1007/s42768-02000041-0, https://doi.org/10.1007/s42768-020-00042-z, https://doi. org/10.1007/s42768-020-00046-9, https://doi.org/10.1007/s42768020-00050-z, https://doi.org/10.1007/s42768-020-00053-w, https:// doi.org/10.1007/s42768-020-00056-7, https://doi.org/10.1007/ s42768-020-00057-6, https://doi.org/10.1007/s42768-020-00061w, https://doi.org/10.1007/s42768-020-00063-8.

Waste Disposal \& Sustainable Energy Editorial Office wdse@zju.edu.cn

1 Zhejiang University Press, Hangzhou 310027, Zhejiang, China

\begin{abstract}
https://doi.org/10.1007/s42768-019-00005-z, https://doi.org/10.1007/s42768-019-00007-x, https://doi.org/10.1007/s42768-019-00008-w, https://doi.org/10.1007/s42768-019-00009-9, https://doi.org/10.1007/s42768-019-00010-2, https://doi.org/10.1007/s42768-019-00011-1, https://doi.org/10.1007/s42768-019-00012-0, https://doi.org/10.1007/s42768-019-00013-z, https://doi.org/10.1007/s42768-019-00014-y, https://doi.org/10.1007/s42768-019-00015-x, https://doi.org/10.1007/s42768-019-00017-9, https://doi.org/10.1007/s42768-019-00019-7, https://doi.org/10.1007/s42768-019-00020-0, https://doi.org/10.1007/s42768-019-00022-y, https://doi.org/10.1007/s42768-019-00023-x, https://doi.org/10.1007/s42768-019-00024-w, https://doi.org/10.1007/s42768-019-00025-9, https://doi.org/10.1007/s42768-019-00026-8, https://doi.org/10.1007/s42768-019-00027-7, https://doi.org/10.1007/s42768-019-00028-6, https://doi.org/10.1007/s42768-019-00029-5, https://doi.org/10.1007/s42768-019-00030-y, https://doi.org/10.1007/s42768-019-00031-x, https://doi.org/10.1007/s42768-019-00032-w, https://doi.org/10.1007/s42768-020-00033-0, https://doi.org/10.1007/s42768-020-00035-y, https://doi.org/10.1007/s42768-020-00036-x, https://doi.org/10.1007/s42768-020-00037-w, https://doi.org/10.1007/s42768-020-00038-9, https://doi.org/10.1007/s42768-020-00039-8, https://doi.org/10.1007/s42768-020-00041-0, https://doi.org/10.1007/s42768-020-00042-z, https://doi.org/10.1007/s42768-020-00046-9, https://doi.org/10.1007/s42768-020-00050-z, https://doi.org/10.1007/s42768-020-00053-w, https://doi.org/10.1007/s42768-020-00056-7, https://doi.org/10.1007/s42768-020-00057-6, https://doi.org/10.1007/s42768-020-00061-w, https://doi.org/10.1007/s42768-020-00063-8.
\end{abstract}

Conflict of Interest statements were not included in the published version of the following articles that appeared in previous issues of Waste Disposal \& Sustainable Energy. The statement is: 


\section{Conflict of interest}

The authors declare that there is no conflict of interest.
Publisher's Note Springer Nature remains neutral with regard to jurisdictional claims in published maps and institutional affiliations. 